\title{
The Urgent Need to Optimize Gestational Weight in Overweight/Obese Women to Lower Maternal- Fetal Morbidities: A Retrospective Analysis on 59,000 Singleton Term Pregnancies
}

\author{
Pierre-Yves Robillard ${ }^{1,2 *}$, Gustaaf Dekker ${ }^{3}$, Malik Boukerrou ${ }^{2,4}$, Brahim Boumahni ${ }^{1}$, Thomas C Hulsey ${ }^{5}$ and Marco Scioscia $^{6}$ \\ ${ }^{1}$ Service de Néonatologie, Centre Hospitalier Universitaire Sud Réunion, Saint-Pierre Cedex, La Réunion, France \\ ${ }^{2}$ Centre d'Etudes Périnatales Océan Indien (CEPOI), Centre Hospitalier Universitaire Sud Réunion, Saint-Pierre cedex, La réunion, France \\ ${ }^{3}$ Department of Obstetrics \& Gynaecology, University of Adelaide, Robinson Institute, Lyell McEwin Hospital, Australia \\ ${ }^{4}$ Service de Gynécologie et Obstétrique, Centre Hospitalier Universitaire Sud Réunion, BP 350, 97448 Saint-Pierre cedex, La réunion, France \\ ${ }^{5}$ Department of epidemiology, School of Public Health, West Virginia University, Morgantown WV, USA \\ ${ }^{6}$ Department of Obstetrics and Gynaecology, Policlinico of Abano Terme, Abano Terme (PD), Italy
}

*Corresponding author: Pierre-Yves Robillard, Service de Néonatologie, Centre Hospitalier Universitaire Sud Reunion, BP 350, 97448 Saint-Pierre Cedex, France; Tel: (262) 2623591 49; Email: robillard.reunion@wanadoo.fr

Received: June 12, 2020; Accepted: June 19, 2020; Published: June 24, 2020

\begin{abstract}
Objective: We retrospectively did a simulation applying the optimal gestational weight gain (optGWG) equation (that we have proposed in 2018) on our population, and observed if its effect on maternal/fetal morbidities in singleton term pregnancies ( $\geq 37$ weeks).
\end{abstract}

Design: Retrospective observational study.

Setting: Single large tertiary maternity unit in Reunion Island, Indian Ocean, overseas French department.

Population or sample: All consecutive singleton births delivered at the Centre Hospitalier Universitaire Hospitalier Sud Reunion's maternity. Standardized epidemiological perinatal database.

Methods: Mathematical simulation on a 19-year historical cohort (2001-2019).

Main outcome measures: Five Maternal/fetal morbidities.

Results: Beginning with overweight women, and enlarging the effect with the rise of different obesities (class I to III) and considering maternal prepregnancy BMI (ppBMI), individualized counselling women on their GWG (optimal gestational weight gain, optGWG) lowers significantly maternal/ fetal morbidities: in a logistic regression model among overweight/obese women, with the outcome optGWG, several morbidities have a negative coefficient as independent factors: cesarean-section, birthweight $\geq 4000 \mathrm{~g}$, term preeclampsia, lowering the effect of rising maternal ppBMI per increment of $5 \mathrm{~kg} / \mathrm{m}^{2}$ (coefficient -0.13 ), all $\mathrm{p}<0.001$.

We propose as a prediction to be verified in future prospective studies that a follow-up and counselling since the first prenatal visit should also lower gestational diabetes mellitus rates.

Conclusion: We may have significant health (and cost) benefits by lowering c-section rates, term preeclampsia, macrosomic babies and LGA babies in overweight/obese women and low-birthweights babies in lean women. We may have much to win from reducing weight gain during pregnancy in overweight/obese women. It is urgent to verify and establish in all continents the specific linear-curve of optGWG for each geographic/ethnic area.

Keywords: Pregnancy, Epidemiology, Pre-pregnancy body mass index, Gestational weight gain, Caesarean section, Obesity, Preeclampsia

\section{Introduction}

Based on a simple axiom: "what is the optimal gestational weight (optGWG) in women to achieve in term pregnancies the natural rate of $10 \%$ of SGA (small for gestational age) as well as 10\% of LGA (Large for gestational age) in newborns", we have found in our population that it is a mathematical linear equation: opGWG $(\mathrm{kg})=-1.2 \mathrm{ppBMI}$ $\left(\mathrm{Kg} / \mathrm{m}^{2}\right)+42 \pm 2 \mathrm{~kg}[1,2]$.
As a matter of fact, when we plot on a graph maternal prepregnancy BMI (ppBMI), and the babies' percentiles, 10\% SGALGA $10 \%$ is materialized by a crossing point. The fact that this $10 \%$ corresponds to a given maternal BMI category suggests that there is a biological maternal-foetal connection. We proposed to call this crossing point the Maternal-Fetal Corpulence Symbiosis (MFCS) [1]. 
Also, since it is a mathematical linear equation it allows that all single women may be considered as a single plot and that we may calculate for each woman at the beginning of pregnancy the individualized optGWG for that pregnancy. Analysing our 19-year cohort, we applied our linear equation on this study population, looking if our proposed optGWG would have changed important outcomes in our population (mathematical simulation).

The purpose of this study is, first, to collect what have been several important maternal/fetal morbidities in our term pregnancies during this 19-year clinical experience: rates of cesarean section, term preeclampsia, gestational diabetes mellitus in women, rates of SGA, LGA, macrosomia $(\geq 4 \mathrm{~kg})$, low birthweights $(<2500 \mathrm{~g})$ and transfers in the neonatal department of newborns. Second, to make a simulation of what would have happened in women with optGWG (optimal weight gain $\pm 2 \mathrm{~kg}$ ) and those with moderately insufficient or excessive GWG ( $\pm 3-9 \mathrm{~kg}$ as compared with optGWG) or severely insufficient or excessive GWG ( $\pm 10 \mathrm{~kg}$ as compared with optGWG).

\section{Material and Methods}

From January $1^{\text {st }}, 2001$, to December 31st, 2019, the hospital records of all women who gave birth at the maternity of the University South Reunion Island were abstracted in a standardized fashion. The study sample was drawn from the hospital perinatal database which prospectively records data of all mother-infant pairs since 2001 . Information is collected at the time of delivery and at the infant hospital discharge and regularly audited by appropriately trained staff. This epidemiological perinatal data base contains information on obstetrical risk factors, description of delivery, and maternal and neonatal outcomes. For the purpose of this study, records have been validated and have been used anonymously. All pregnant women in Reunion Island as part of the French National Health Care System have their prenatal visits, biological and ultasonographic examinations, and anthropological characteristics recorded in a maternity booklet.

Preeclampsia, gestational hypertension and eclampsia were diagnosed according to the definition issued by the International Society for the Study of Hypertension in Pregnancy (ISSHP) relatively to the guidelines in force at the year of pregnancy. In the present study, because optimal weight gain has been described only for term pregnancies [1], we have selected only women who delivered live births at term (37-42 weeks).

\section{Design and Study Population}

The maternity department of Saint Pierre hospital is a tertiary care centre that performs about 4,300 deliveries per year, thus representing about $85 \%$ of deliveries of the Southern area of Reunion Island, and it is the only level-3 maternity (the other maternity is a private clinic). Reunion Island is a French overseas region in the Southern Indian Ocean. The entire pregnant population has access to maternity care free of charge as provided by the French healthcare system, which combines freedom of medical practice with nationwide social security. Prenatal system is based on scheduled appointments (9 prenatal visits and on average 4 ultrasounds) starting from 6 to 8 (see below) weeks of gestation.

\section{Definition of Exposure and Outcomes}

Booking BMI (ppBMI), was obtained at the first antenatal visit (average 6-8 weeks). Women are systematically weighted at their arrival in labour \& delivery. In rare cases of imminent delivery $(<$ $10 \%$ ) the documented weight during the last antenatal visit prior to birth was used for calculations.

\section{Primary Outcome}

We arbitrarily created 5 categories of GWG using the published formula:

(optGWG $=-1.2 \mathrm{x}+42 \mathrm{ppBMI}-\mathrm{kg} / \mathrm{m}^{2}- \pm 2 \mathrm{~kg}$ ) [1] defined in our population of Reunion island.

- Optimal GWG range: optimal GWG result PLUS or MINUS $2 \mathrm{~kg}$ (the formula).

- Insufficient GWG:

- Moderately insufficient: adequate GWG minus 3 to minus 9 $\mathrm{kg}$.

- Severely insufficient: adequate GWG minus $10 \mathrm{~kg}$ and below:

- Excessive GWG:

- Moderately excessive: adequate GWG PLUS 3 to plus $9 \mathrm{~kg}$.

- Severely excessive: adequate GWG PLUS $10 \mathrm{~kg}$ and over.

- Screening of GDM is systematically made in all pregnant women in the first trimester: until 2016 it was the O'Sullivan test (50g glucose, blood glucose level after 1 hour). The threshold for hyperglycemia being $1.4 \mathrm{~g} / \mathrm{l}$. Since 2016, this test has been replaced in all women by a fasting glycemia in the first trimester, the threshold for positivity being 0.92 $\mathrm{g} / \mathrm{l}$. As the incidence of gestational diabetes mellitus is very high in Reunion GTT is made (between 24-28 weeks) to ALL pregnant women (even if they have a normal 1st trimester blood glucose). Those who have no GTT are only those who have a $1^{\text {st }}$ trimester blood glucose over $1.26 \mathrm{~g} / \mathrm{l}$, these last being considered as Type 2 diabetes.

\section{Statistical Analysis}

- Data is presented as numbers and proportions (\%) for categorical variables and as mean and Standard Deviation (SD) for continuous ones. Comparisons between groups were performed by using $\chi^{2}$-test; Odds Ratio (OR) with $95 \%$ Confidence Interval (CI) was also calculated. Paired t-test was used for parametric and the Mann-Whitney $U$ test for non-parametric continuous variables. P-values $<0.05$ were considered statistically significant. Epidemiological data have been recorded and analysed with the software EPI-INFO 7.1.5 (2008, CDC Atlanta, OMS), EPIDATA 3.0 and EPIDATA Analysis V2.2.2.183. Denmark.

\section{Ethnic Origin}

Reunionese women comprise a melting pot of African and African intermixed populations for ap. $82 \%$ of the inhabitants (the $18 \%$ other 
Pierre-Yves Robillard (2020) The Urgent Need to Optimize Gestational Weight in Overweight/Obese Women to Lower Maternal-Fetal Morbidities: A Retrospective Analysis on 59,000 Singleton Term Pregnancies

being Europeans from mainland France): Dravidian Indian (SouthIndia, Madras and Pondichery) and very few Chinese origin. Therefore "Reunion origin" comprises roughly African intermixed origin for approximately $75 \%$ and Dravidian Indians (South India, Tamils) for 25\%. The French Constitution (and therefore laws) forbids ethnicity, religion or political opinions of the citizens on viral or scientific records. Therefore, we could not include ethnicity in any logistical model.

To validate the independent association of maternal age and other confounding factors on term optimal gestational weight gain (optGWG) we realized a multiple regression logistic model. Variables associated with optGWG in bivariate analysis, with a p-value below 0.1 or known to be associated with the outcome in the literature were included in the model. A stepwise backward strategy was then applied to obtain the final model. The goodness of fit was assessed using the Hosmer-Lemeshow test. A p-value below 0.05 was considered significant. All analyses were performed using MedCalc software (version 12.3.0; MedCalc Software's, Ostend, Belgium).
Optimal gestational weight gain (YES/NO) being the outcome measure, we considered the following covariates as possible confounders in this analysis: pre-pregnancy maternal BMI by increment of $5 \mathrm{~kg} / \mathrm{m}^{2}$, gestational diabetes, caesarean section rates, term preeclampsia, birthweights over $4 \mathrm{~kg}$, and newborn's transfers in the neonatal departments. We included these variables and calculated the $\chi^{2}$ for trend (Mantel extension), the odds ratios for each exposure level compared with the first exposure level.

\section{Results}

During the 19-year period, there were 68,047 term (37-42 weeks) singleton live births in University maternity of South-Reunion Island. We could determine gestational weight gain in 65,738 women $(96.6 \%$ of women) and determine optimal GWG in 59,171 (87\%) of women (requirement to have also the information on mothers' heights to calculate the BMI).

Table 1 shows population characteristics depicts crude results in our population. Pregnancies were well followed (9 prenatal visits, 4.4

Table 1: Population characteristics. Term pregnancies $\geq 37$ weeks gestation with known GWG end of pregnancy N=65,738 ( $96.6 \%$ of the entire cohort). Live births only (and total births for intrauterine fetal deaths).

\begin{tabular}{|c|c|c|c|c|c|}
\hline Characteristics & $\begin{array}{l}\text { All term pregnancies }(\geq 37 \text { weeks }) \\
\qquad \mathrm{N}=65,738(\%)\end{array}$ & $\begin{array}{l}\text { Women with optimal weight gain } \\
\qquad \mathrm{N}=12,594\end{array}$ & $\begin{array}{l}\text { Other women "reference" } \\
\mathrm{N}=55,453 \\
\end{array}$ & $\begin{array}{c}\text { OR [95\% CI] Optimal GWG } \\
\text { vs. reference } \\
\end{array}$ & p-value \\
\hline Maternal age (SD) & $27.7 \pm 6.5$ & $27.7 \pm 6.5$ & $28.0 \pm 6.5$ & Difference 0.3 year & 0.001 \\
\hline Parity \pm sd & $1.28 \pm 1.5$ & $1.24 \pm 1.5$ & $1.28 \pm 1.5$ & & 0.03 \\
\hline Primiparity & $25,297(37.2)$ & $4655(37.0)$ & $20,642(37.2)$ & & 0.58 \\
\hline Women living single & $24,528(36.2)$ & $4309(34.3)$ & $20,219(36.6)$ & & \\
\hline Education $>10$ years & $38,466(58.1)$ & $7310(59.7)$ & $31,156(58.3)$ & $\begin{array}{c}1.06 \\
{[1.02-1.1]} \\
\end{array}$ & 0.005 \\
\hline Origin Reunion Island & $55,700(82.2)$ & $10,403(82.8)$ & $45,297(81.9)$ & & NS \\
\hline BMI $\left(\right.$ mean $\left.\pm \mathrm{sd}, \mathrm{kg} / \mathrm{m}^{2}\right)$ & $\begin{array}{l}24.7 \pm 5.9 \\
\mathrm{~N}=65,738\end{array}$ & $\begin{array}{l}24.4 \pm 4.3 \\
\mathrm{~N}=12,592\end{array}$ & $\begin{array}{l}24.8 \pm 6.3 \\
\mathrm{~N}=53,146\end{array}$ & $\begin{array}{l}\text { Difference } \\
0.4 \mathrm{~kg} / \mathrm{m}^{2}\end{array}$ & $<0.0001$ \\
\hline Obesity $\geq 30 \mathrm{~kg} / \mathrm{m}^{2}$ & $11,547(17.6)$ & $1418(11.3)$ & $10,129(19.1)$ & $\begin{array}{c}0.54 \\
{[0.51-0.57]} \\
\end{array}$ & $<0.0001$ \\
\hline \multicolumn{6}{|l|}{ BMI categories } \\
\hline - $\quad \leq 19$ (underweight) & $13,713(20.8)$ & $1589(12.6)$ & $12,124(22.6)$ & $0.51[0.48-0.55]$ & $<0.0001$ \\
\hline - $\quad 20-24$ (normal) & $26,294(40.0)$ & $6032(47.9)$ & $20,262(38.1)$ & $1.6[1.53-1.66]$ & $<0.0001$ \\
\hline - $\quad$ 25-29 overweight & $14,184(21.6)$ & $3553(28.2)$ & $10,631(20.0)$ & $1.7[1.58-1.73]$ & $<0.0001$ \\
\hline - $\quad 30-34$ (obesity I) & $7017(10.7)$ & $1175(9.3)$ & $5842(11.0)$ & $0.87[0.82-0.93]$ & $<0.0001$ \\
\hline - $\quad 35-39$ (obesity II) & $3021(4.6)$ & $205(1.6)$ & $2816(5.3)$ & $0.31[0.27-0.35]$ & $<0.0001$ \\
\hline - $\quad>40$ (obesity III) & $1509(2.2)$ & $38(0.3)$ & $1471(2.8)$ & $0.11[0.08-0.15]$ & $<0.0001$ \\
\hline Smoking & $8205(12.1)$ & $1880(11.8)$ & $6725(12.1)$ & 0.96 & 0.25 \\
\hline $\mathrm{Nb}$ of prenatal visits & $9.0 \pm 2.73$ & $9.1 \pm 2.62$ & $8.9 \pm 2.74$ & & 0.001 \\
\hline Number of ultrasonographies & $4.4 \pm 1.7$ & $4.4 \pm 1.5$ & $4.4 \pm 1.7$ & 0.99 & 0.93 \\
\hline Weight gain (kg) & $\begin{array}{l}12.1 \pm 6.2 \\
\mathrm{~N}=65,738\end{array}$ & $\begin{array}{l}12.6 \pm 5.1 \\
\mathrm{~N}=12,592\end{array}$ & $\begin{array}{l}11.9 \pm 6.5 \\
\mathrm{~N}=47,798 \\
\end{array}$ & Difference $0.7 \mathrm{~kg}$ & $<0.0001$ \\
\hline Gestational diabetes & $7061(10.8)$ & $1371(11.0)$ & $6103(11.1)$ & 0.99 & 0.64 \\
\hline Chronic hypertension & $902(1.3)$ & $141(1.1)$ & $761(1.4)$ & $0.81[0.68-0.97]$ & 0.02 \\
\hline Term preeclampsia & $760(1.1)$ & $100(0.8)$ & $660(1.2)$ & $0.66[0.54-0.82$ & 0.001 \\
\hline Hospitalization & $7949(11.7)$ & $1422(11.3)$ & $6527(11.8)$ & 0.95 & 0.12 \\
\hline C-section & $9971(14.7)$ & $1478(13.9)$ & $8223(14.8)$ & $0.93[0.88-0.98]$ & 0.007 \\
\hline Induced delivery & $14,979(22.0)$ & $2738(21.7)$ & $12241(22.1)$ & 0.098 & 0.42 \\
\hline Birth weight (g) & $3184 \pm 440$ & $3226 \pm 421$ & $3175 \pm 447$ & Difference $51 \mathrm{~g}$ & $<0.0001$ \\
\hline Low BW $<2500 \mathrm{~g}$ & $3592(5.3)$ & $473(3.8)$ & $3119(5.8)$ & $0.65[0.6-0.7]$ & $<0.0001$ \\
\hline Small for gestational age & $7139(10.5)$ & $1053(8.4)$ & $6086(11.0)$ & $0.74[0.7-0.8]$ & $<0.0001$ \\
\hline Large for gestational age & $6434(9.5)$ & $1248(9.9)$ & $5186(9.4)$ & $1.07[1.0-1.14]$ & 0.05 \\
\hline Birthweight $\geq 4000 \mathrm{~g}$ & $2636(3.9)$ & $473(3.8)$ & $3119(5.6)$ & $0.65[0.59-0.72]$ & $<0.0001$ \\
\hline Neonatal transfers & $2918(4.3)$ & $467(3.7)$ & $2451(4.4)$ & $0.83[0.75-0.92]$ & 0.001 \\
\hline Intrauterine fetal deaths & $115 / 68,179(0.2)$ & $19 / 12611(0.2)$ & $96 / 55,668(0.2)$ & 0.87 & 0.58 \\
\hline
\end{tabular}


Pierre-Yves Robillard (2020) The Urgent Need to Optimize Gestational Weight in Overweight/Obese Women to Lower Maternal-Fetal Morbidities: A Retrospective Analysis on 59,000 Singleton Term Pregnancies

ultrasonographies in average), our population is young (27 years in average), with a high rate of women declaring living single (36\%), and a high rate of obesity $\left(\geq 30 \mathrm{~kg} / \mathrm{m}^{2}, 17.6 \%\right)$.

When we look at crude results of BMI categories, it is of note (second column, women with optGWG) that only women with normal BMI $\left(20-24.9 \mathrm{~kg} / \mathrm{m}^{2}\right)$ reached an acceptable score of optGWG in $48 \%$ of cases. For overweight women $\left(25-29.9 \mathrm{~kg} / \mathrm{m}^{2}\right), 28 \%$ of cases only. For underweight women $<19 \mathrm{~kg} / \mathrm{m}^{2}$, only $12 \%$. For obese and severe obese women, it is worse: $9 \%$ to $1 \%$.

Comparing maternal/foetal morbidities, in these crude results the compared Odds-ratios between "optGWG" vs. "reference" (those who did not achieve optGWG $\pm 2 \mathrm{~kg}$ ): C-section rate OR 0.93 [0.880.98 ], $\mathrm{p}=0.007$, term preeclampsia OR 0.66 [0.54-0.82], $\mathrm{p}=0.001$, low birthweights $<2500 \mathrm{~g}$, OR 0.65 [0.6-0.7], $\mathrm{p}=0.0001$, birthweights $\geq 4000 \mathrm{~g}$, OR 0.65 [0.59-0.72], $\mathrm{p}=0.0001$, neonatal transfers OR 0.83 [0.75-0.92], $\mathrm{p}=0.001$.

Tables 2-5, with known GWG and ppBMI (information on height), $\mathrm{N}=59,171$ ( $87 \%$ of the entire cohort) detail the GWG for non-obese women $\left(<25 \mathrm{~kg} / \mathrm{m}^{2}\right)$, overweight $(25-29.9)$, obese class I $\left(30-34.9 \mathrm{~kg} / \mathrm{m}^{2}\right)$ and severe obese $\left(35 \mathrm{~kg} / \mathrm{m}^{2}\right.$ and over). We categorized in optGWG, moderately insufficient or excessive optGWG $\pm 3-9 \mathrm{~kg}$, severely insufficient or excessive optGWG $\pm 10 \mathrm{~kg}$.

Table 2: Simulation from the perspective of optimal or non-optimal GWG. Rates (\%) of several maternal/foetal morbidities. Non obese women $<25 \mathrm{~kg} / \mathrm{m}^{2}$. N=36,167.

\begin{tabular}{|c|c|c|c|c|c|c|c|}
\hline $\begin{array}{l}\text { Differences with adequate } \\
\text { Weight gain Non-obese women }\end{array}$ & C-section rate (\%) & $\begin{array}{c}\text { Term preeclampsia } \\
(\%)\end{array}$ & $\begin{array}{c}\text { Gestational } \\
\text { diabetes }\end{array}$ & SGA (\%) & LGA (\%) & $\mathrm{BW} \geq 4 \mathrm{~kg}(\%)$ & $\begin{array}{c}\text { Neonatal Transfers } \\
(\%) \\
\end{array}$ \\
\hline$-10 \mathrm{~kg}$ and lower $\mathrm{N}=4518$ & $384(8.5)$ & $20(0.4)$ & $295(6.5)$ & $1028(22.8)$ & $89(2.0)$ & $24(0.5)$ & $181(4.0)$ \\
\hline$-3-9 \mathrm{~kg}, \mathrm{~N}=18,121$ & $1921(10.6)$ & $103(0.6)$ & $1167(6.4)$ & $2161(11.9)$ & $1034(5.7)$ & $316(1.7)$ & $573(3.2)$ \\
\hline Adequate GWG $\pm 2 \mathrm{~kg}, \mathrm{~N}=7621$ & $985(12.9)$ & $67(0.9)$ & $438(5.8)$ & $629(8.3)$ & $714(9.4)$ & $270(3.5)$ & $270(3.5)$ \\
\hline$+3-9 \mathrm{~kg} . \mathrm{N}=5187$ & $786(15.2)$ & $72(1.4)$ & $243(4.7)$ & $302(5.8)$ & $649(12.5)$ & $331(6.4)$ & $204(3.9)$ \\
\hline $10 \mathrm{~kg}+\mathrm{N}=703$ & $125(17.8)$ & $24(3.4)$ & $46(6.6)$ & $41(5.8)$ & $133(18.9)$ & $81(11.5)$ & $31(4.4)$ \\
\hline Observed rates. $\mathrm{N}=36,167$ & $4201(11.6)$ & $286(0.8)$ & $2189(6.1)$ & $4161(11.5)$ & $2619(7.2)$ & $1022(2.8)$ & $1259(3.5)$ \\
\hline $\begin{array}{l}\text { Odds Ratios: "Adequate" vs. } \\
\text { observed }\end{array}$ & $\begin{array}{c}1,12[1.04-1.2] \\
\mathrm{P}=0.001\end{array}$ & $1.11 \mathrm{P}=0.21$ & $0.94 \mathrm{P}=0.15$ & $\begin{array}{c}0.69[0.63-0.75] \\
\mathrm{P}=0.001\end{array}$ & $\begin{array}{c}1.32[1.2-1.4] \\
\mathrm{P}=0,001\end{array}$ & $\begin{array}{c}1.26[1.1-1.4] \\
\mathrm{P}=0.001\end{array}$ & $1.01 \mathrm{P}=0.39$ \\
\hline
\end{tabular}

Table 3: Simulation from the perspective of optimal or non-optimal GWG. Rates (\%) of several maternal/foetal morbidities. Overweight women $25-29.9 \mathrm{~kg} / \mathrm{m}^{2}$. N=12,701.

\begin{tabular}{|c|c|c|c|c|c|c|c|}
\hline $\begin{array}{l}\text { Differences with adequate Weight } \\
\text { gain Overweight } 25-29.9 \mathrm{~kg} / \mathrm{m}^{2}\end{array}$ & $\mathrm{C}$-section rate (\%) & Term preeclampsia (\%) & Gestational diabetes & SGA (\%) & LGA (\%) & $\mathrm{BW} \geq 4 \mathrm{~kg}(\%)$ & $\begin{array}{c}\text { Neonatal Transfers } \\
(\%)\end{array}$ \\
\hline$-10 \mathrm{~kg}$ and lower $\mathrm{N}=267$ & $22(8.2)$ & $0(0.0)$ & $52(19.6)$ & $51(19.1)$ & $89(2.0)$ & $11(4.1)$ & $9(3.4)$ \\
\hline$-3-9 \mathrm{~kg}, \mathrm{~N}=2877$ & $382(13.3)$ & $25(0.9)$ & $519(18.2)$ & $365(12.7)$ & $1034(5.7)$ & $186(6.5)$ & $91(3.2)$ \\
\hline Adequate $G W G \pm 2 \mathrm{~kg}, \mathrm{~N}=3553$ & $522(14.7)$ & $22(0.6)$ & $577(16.4)$ & $294(8.3)$ & $714(9.4)$ & $377(10.6)$ & $145(4.1)$ \\
\hline$+3-9$ kg. $\mathrm{N}=4736$ & $847(17.9)$ & $66(1.4)$ & $562(12.0)$ & $327(6.9)$ & $649(12.5)$ & $642(13.6)$ & $186(3.9)$ \\
\hline $10 \mathrm{~kg}+\mathrm{N}=1268$ & $288(22.7)$ & $33(2.6)$ & $120(9.6)$ & $78(6.2)$ & $133(18.9)$ & $245(19.3)$ & $61(4.8)$ \\
\hline Observed rates. $\mathrm{N}=12,701$ & $2061(16.2)$ & $146(1.2)$ & $1830(14.6)$ & $1115(8.8)$ & $2619(7.2)$ & $1461(11.5)$ & $492(3.9)$ \\
\hline Odds Ratios: "Adequate" vs. observed & $\begin{array}{c}0.88[0.79-0.98] \\
\mathrm{P}=0.01\end{array}$ & $0.53[0.33-0.83] \mathrm{P}=0.003$ & $1.15[1.04-1.3] \mathrm{P}=0.003$ & $0.93 \mathrm{P}=0.17$ & $0.96 \mathrm{P}=0.24$ & $0.91 \mathrm{P}=0.07$ & $0.91 \mathrm{P}=0.28$ \\
\hline
\end{tabular}

Table 4: Simulation from the perspective of optimal or non-optimal GWG. Rates (\%) of several maternal/foetal morbidities. Obesity class I: $30-34.9 \mathrm{~kg} / \mathrm{m}^{2}$. N=6232.

\begin{tabular}{|c|c|c|c|c|c|c|c|}
\hline \begin{tabular}{|l|} 
Differences with adequate Weight \\
gain Obese I $30-34.9 \mathrm{~kg} / \mathrm{m}^{2}$
\end{tabular} & C-section rate (\%) & Term preeclampsia (\%) & Gestational diabetes & SGA (\%) & LGA (\%) & $\mathrm{BW} \geq 4 \mathrm{~kg}(\%)$ & $\begin{array}{c}\text { Neonatal. } \\
\text { Transfers (\%) }\end{array}$ \\
\hline$-10 \mathrm{~kg}$ and lower $\mathrm{N}=73$ & $11(15.1)$ & $0(0.0)$ & $18(25.0)$ & $11(15.1)$ & $2(2.7)$ & $1(1.4)$ & $5(6.8)$ \\
\hline$-3-9 \mathrm{~kg}, \mathrm{~N}=561$ & $85(15.2)$ & $4(0.7)$ & $152(27.3)$ & $70(12.5)$ & $39(7.0)$ & $14(2.5)$ & $21(3.7)$ \\
\hline Adequate GWG $\pm 2 \mathrm{~kg}, \mathrm{~N}=1175$ & $198(16.9)$ & $9(0.8)$ & $279(24.1)$ & $111(9.4)$ & $136(11.6)$ & $53(4.5)$ & $48(4.1)$ \\
\hline$+3-9$ kg. $\mathrm{N}=2898$ & $623(21.5)$ & $47(1.6)$ & $582(20.5)$ & $228(7.9)$ & $413(14.3)$ & $151(5.2)$ & $107(3.7)$ \\
\hline $10 \mathrm{~kg}+\mathrm{N}=1525$ & $364(23.9)$ & $43(2.8)$ & $223(15.0)$ & $109(7.1)$ & $293(19.2)$ & $150(9.8)$ & $72(4.7)$ \\
\hline Observed rates. $\mathrm{N}=6232$ & $1281(20.6)$ & $103(1.7)$ & $1254(20.5)$ & $529(8.5)$ & $883(14.2)$ & $369(5.9)$ & $253(4.1)$ \\
\hline Odds Ratios: "Adequate" vs. observed & $\begin{array}{c}0.78[0.66-0.92] \\
\mathrm{P}=0.002\end{array}$ & $0.45[0.22-0.87] \mathrm{P}=0.01$ & $1.23[1.06-1.4] \mathrm{P}=0.002$ & $1.12 \mathrm{P}=0.14$ & $\begin{array}{c}0.79[0.65-0.96] \\
P=0.009\end{array}$ & $\begin{array}{c}0.75[0.55-1.0] \\
P=0.03\end{array}$ & $1.0 \mathrm{P}=0,48$ \\
\hline
\end{tabular}

Table 5: Simulation from the perspective of optimal or non-optimal GWG. Rates (\%) of several maternal/foetal morbidities. Severe Obesity $\geq 35 \mathrm{~kg} / \mathrm{m}^{2}$. N=4071.

\begin{tabular}{|c|c|c|c|c|c|c|c|}
\hline $\begin{array}{l}\text { Differences with adequate Weight } \\
\text { gain Obese I } 30-34.9 \mathrm{~kg} / \mathrm{m}^{2}\end{array}$ & C-section rate $(\%)$ & Term preeclampsia (\%) & Gestational diabetes & SGA (\%) & LGA (\%) & $\mathrm{BW} \geq 4 \mathrm{~kg}(\%)$ & $\begin{array}{c}\text { Neonatal. Transfers } \\
(\%)\end{array}$ \\
\hline$-10 \mathrm{~kg}$ and lower $\mathrm{N}=16$ & $1(6.3)$ & $0(0.0)$ & $5(33.3)$ & $3(18.8)$ & $1(6.3)$ & $1(6.3)$ & $0(0.0)$ \\
\hline$-3-9 \mathrm{~kg}, \mathrm{~N}=106$ & $13(12.3)$ & $2(1.9)$ & $37(35.2)$ & $14(13.2)$ & $9(8.5)$ & $3(2.8)$ & $6(5.7)$ \\
\hline Adequate GWG $\pm 2 \mathrm{~kg}, \mathrm{~N}=243$ & $43(17.7)$ & $2(0.8)$ & $77(32.5)$ & $19(7.8)$ & $21(8.6)$ & $5(2.1)$ & $4(1.6)$ \\
\hline$+3-9$ kg. $N=1300$ & $276(21.2)$ & $24(1.8)$ & $357(28.0)$ & $113(8.7)$ & $164(12.6)$ & $61(4.7)$ & $66(5.1)$ \\
\hline $10 \mathrm{~kg}+\mathrm{N}=2406$ & $655(27.2)$ & $76(3.2)$ & $608(26.3)$ & $144(6.0)$ & $492(20.4)$ & $229(9.5)$ & $139(5.8)$ \\
\hline Observed rates. $\mathrm{N}=4071$ & $988(24.3)$ & $104(2.6)$ & $1084(27.5)$ & $293(7.2)$ & $687(16.9)$ & $299(7.3)$ & $215(5.3)$ \\
\hline Odds Ratios: "Adequate" vs. observed & $\begin{array}{c}0.67[0.47-0.93] \\
P=0.009\end{array}$ & $0.31[0.05-1.07] \mathrm{P}=0.07$ & $1.27[0.97-1.7] \mathrm{P}=0.06$ & $1.09 \mathrm{P}=0.40$ & $\begin{array}{c}0.46[0.29-0.73] \\
P=0.001\end{array}$ & $\begin{array}{c}0.46[0.1-0.6] \\
P=0.001\end{array}$ & $\begin{array}{c}0.30[0.1-0.74] \\
\mathrm{P}=0.006\end{array}$ \\
\hline
\end{tabular}


Pierre-Yves Robillard (2020) The Urgent Need to Optimize Gestational Weight in Overweight/Obese Women to Lower Maternal-Fetal Morbidities: A Retrospective Analysis on 59,000 Singleton Term Pregnancies

For all the tables (first column on the left) we observed in our population during this 19 -year period a majority of women considered by our proposed equation as inadequate GWG:

For Table 2, non obese: $63 \%$ in categories of INSUFFICIENT GWG $(18,121+4518 / 36,167)$.

For Table 3, overweight: $47 \%$ in categories of EXCESSIVE GWG $(4736+1268 / 12,701)$.

For Table 4, Obese class I: 71\% in categories of EXCESSIVE GWG $(2898+1525 / 6232)$.

For Table 5, Severe obese: 91\% in categories of EXCESSIVE GWG $(1300+2406 / 4071)$.

In all the Tables 2 to 5 the calculated crude odds-ratios are comparisons between optGWG and the observed rate in the total population (and not with some categories of inadequate GWG). These OR may be easily calculated as much higher if compared with for example the $71 \%$ of excessive weight gain seen in obese class I (30$34.9 \mathrm{~kg} / \mathrm{m}^{2}$ ) and optGWG.

Table 2 (non obese women), $\mathrm{N}=36,167$ women ( $61 \%$ of our population): For this "normal" population, comparisons with optimal GWG did not give important differences with actually happened during the 19 year clinical practice. It is of note that the optGWG women had 10\% more C-sections (OR 1.12, p = 0.001), LGA babies (9.4\% vs. 7.2$)$ and birthweights over $4 \mathrm{~kg}$ (3.5\% vs. $2.8 \%)$

Table 3 (overweight women), $\mathrm{N}=12,701$ women: OptGWG women have significantly less caesarean section rate (OR $0.88, \mathrm{p}=$ 0.01 ), less term preeclampsia (OR $0.53, p=0.003$, and a tendency to have less newborns with birthweights over $4 \mathrm{~kg}(\mathrm{OR} 0.91, \mathrm{p}=0.07)$.

Table 4 obesity class I and Table 5 severe obese $\left(\geq 35 \mathrm{~kg} / \mathrm{m}^{2}\right)$ : All crude comparisons for the chosen morbid items are statistically significant: OptGWG women had less caesarean section rate (respectively OR 0.78 and $0.67, \mathrm{p}=0.002$ ), less term preeclampsia (OR $0.45, \mathrm{p}=0.01$ and $0.31 \mathrm{p}=0.07$ ), less LGA (OR 0.79 and $0.46, \mathrm{p}=$ $0.001)$, less birthweights over $4 \mathrm{~kg}$ (OR $0.75, \mathrm{p}=0.03$ and $0.46, \mathrm{p}=$ $0.001)$ and less neonatal transfers in neonatology for severe obese $(\geq$ $\left.35 \mathrm{~kg} / \mathrm{m}^{2}\right)$ : OR $0.30, \mathrm{p}=0.006$.

It is of special note that in overweight and all obese women (Tables 3-5) incidence of GDM is higher in optGWG women than in the observed rate.
Table 6 As the effect of achieving optimal weight gain is largely concentrated in overweight and all kind of obesities, we performed our logistic model only in overweight and obese class I,II and III women (therefore the 25,731 pregnancies with pre-pregnancy BMI $\geq 25 \mathrm{~kg} /$ $\mathrm{m}^{2}$ ). Multiple logistic regression model to validate the independent association of optimal GWG with different maternal-foetal morbidities. Controlling for all the other variables, several morbidities have a negative coefficient as independent factors: cesarean-section (coefficient -0.20 , decrease of $20 \%$ ), birthweight $\geq 4000 \mathrm{~g}$ (coefficient -0.38), term preeclampsia (coefficient -0.79 ), maternal overweight pre-pregnancy BMI (coefficient -0.13 , decrease of $13 \%$ of the BMI effect per increment of $5 \mathrm{~kg} / \mathrm{m}^{2}$ using optimal weight gain). However, adequate GWG have a positive coefficient with GDM: 0.19, increase of the risk by $19 \%$.

Figure 1 shows simulation based on maternal pre-pregnancy BMI by increments of $5 \mathrm{~kg} / \mathrm{m}^{2}$ (upper case) maternal morbidities C-sections and term preeclampsia rates and (lower case) neonatal morbidities birthweight over $4 \mathrm{~kg}$ and transfers in neonatal department. These figures consider all the spectrum of maternal pre-pregnancy BMI from lean women to obesity class III. In dark lines, the 19 yearexperience observed rates, in dash lines the calculated rates if women had achieved the optGWG (window of $4 \mathrm{~kg}$ specific to each woman). Visually, we can estimate the strong effect of optGWG beginning with overweight BMI and emphasized with rising BMI.

Figure 2 shows simulation of optimal or non-optimal GWG for all categories of ppBMI (lean to obese). We can visualize the effect of insufficient or excessive GWG on the outcomes LGA, macrosomic babies, SGA and C-sections rates.

Not shown in the Tables and Figures: the rate of low birthweight newborns $(<2500 \mathrm{~g})$. In non obese women $\left(<25 \mathrm{~kg} / \mathrm{m}^{2}\right)$, it is of $3.7 \%$ in optGWG vs. $5.8 \%$ in observed rates OR 0.64 [0.57-0.73], p $<0.001$ women. Respectively in overweight $3.9 \%$ vs. $4.3 \%$ (NS), class I obese $4.1 \%$ vs. $3.7 \%(\mathrm{NS})$, in severely obese women $\left(\geq 35 \mathrm{~kg} / \mathrm{m}^{2}\right) 3.7 \%$ vs. $3.7 \%$.

\section{Discussion}

Our calculations on simulated maternal/fetal morbidities in our term pregnancies, (rates of cesarean section, term preeclampsia, GDM, SGA, LGA, macrosomia ( $\geq 4 \mathrm{~kg}$ ), low birthweights $(<2500 \mathrm{~g}$ ) and transfers in the neonatal department of newborns) demonstrate that achieving "Maternal Fetal Corpulence Symbiosis, MFCS" [1] in

Table 6: Outcome: optimal gestational weight gain in overweight/obese women (pre-pregnancy BMI $\geq 25 \mathrm{~kg} / \mathrm{m}^{2}, \mathrm{~N}=25,731$ pregnancies). Multiple logistic regression model to validate the independent association of optimal GWG with different maternal-foetal morbidities. Controlling for all the other variables, several morbidities have a negative coefficient as independent factors: cesarean-section (coefficient -0.20 , decrease of $20 \%$ ), birthweight $\geq 4000 \mathrm{~g}$ (coefficient -0.38 ), term preeclampsia (coefficient -0.79 ), maternal overweight pre-pregnancy BMI (coefficient -0.13 , decrease of $13 \%$ of the BMI effect per increment of $5 \mathrm{~kg} / \mathrm{m}^{2}$ using optimal weight gain). However, adequate GWG have a positive coefficient with GDM: 0.19 , increase of the risk by some $20 \%$.

\begin{tabular}{|c|c|c|c|c|}
\hline & \multicolumn{4}{|c|}{$\begin{array}{l}\text { Multiple Logistic Regression } \\
\text { Outcome: optimal gestational weight gain }\end{array}$} \\
\hline & Coefficient & Odds Ratio & $95 \% \mathrm{CI}$ & $\mathbf{P}$ \\
\hline Cesarean section & -0.20 & 0.81 & {$[0.74-0.89]$} & $<0.0001$ \\
\hline Birthweight $\geq 4000 \mathrm{~g}$ & -0.38 & 0.67 & {$[0.57-0.79]$} & $<0.0001$ \\
\hline Pre pregnancy maternal BMI (increment of $5 \mathrm{~kg} / \mathrm{m}^{2}$ ) & -0.13 & 0.87 & {$[0.86-0.88]$} & $<0.0001$ \\
\hline Gestational diabetes mellitus & 0.19 & 1.21 & [1.11-1.31] & $<0.0001$ \\
\hline Term preeclampsia & -0.79 & 0.45 & {$[0.31-0.65]$} & $<0.0001$ \\
\hline Transfer in neonatal department & -0.09 & 0.90 & {$[0.77-1.06]$} & 0.25 \\
\hline
\end{tabular}


Pierre-Yves Robillard (2020) The Urgent Need to Optimize Gestational Weight in Overweight/Obese Women to Lower Maternal-Fetal Morbidities: A Retrospective Analysis on 59,000 Singleton Term Pregnancies
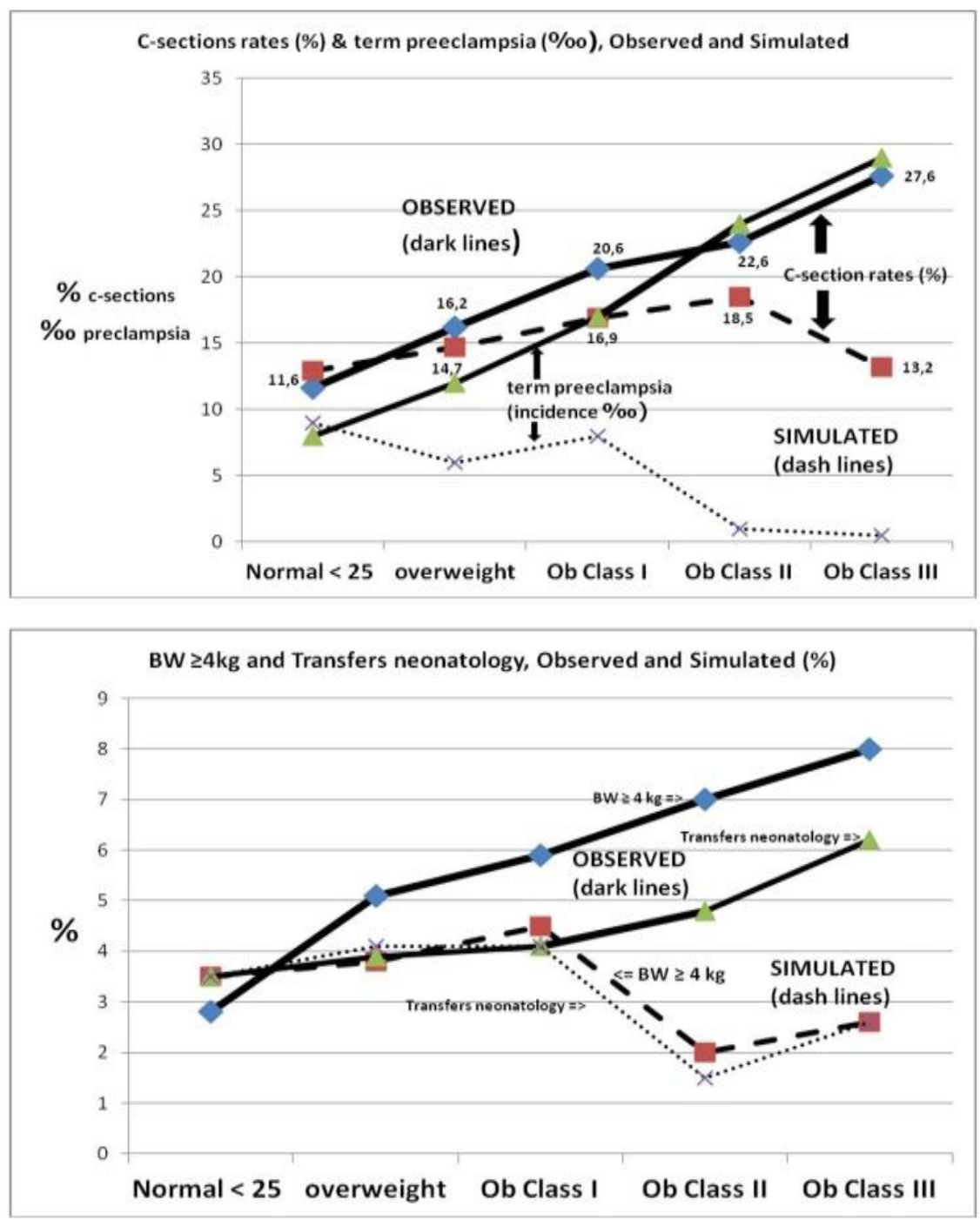

Figure 1: Simulation based on maternal pre-pregnancy BMI by increments of $5 \mathrm{~kg} / \mathrm{m}^{2}$. Maternal and neonatal morbidities. Dark lines: observed rates, in dash lines the calculated rates if women had achieved the optGWG (window of $4 \mathrm{~kg}$ specific to each woman).

all women would have the potential to significantly lower important maternal/fetal morbidities, except, surprisingly and for now, the rate of GDM.

We have put an online calculator consultable on smart phone at REPERE.RE (REseau PErinatal REunion), in three languages (French, Spanish and English) [2], and any reader is invited to validate these findings in their own populations.

It is of note that achieving MFCS, our rates of SGA-LGA in all the tables and figures reproduced in this paper, we notice that the equilibrium points (optGWG) show the closest combination to the $10 \%$ SGA/LGA crossing point. First, concerning the huge debates on gestational weight gain and maternal obesity (class I-III), we will not in this paper recall all these controversies (and the numerous critics, for example, those made by our Asian colleagues [3], China, Korea, Japan, India on the current IOM 2009 recommendations [4]) as they have been recently extensively discussed by ourselves elsewhere $[1,5,6]$.
Second, the "gestational diabetes mellitus paradox" it is noteworthy in all the tables to find an inverse relationship between optimal GWG and the risk of gestational diabetes. Lowering the GWG seems to heighten the risk of GDM, and excessive weight gain to lower it! GDM seems to be the only significant maternal risk that is not ameliorated by achieving an optGWG. In Table 6 , the logistic regression model, controlling for other risks, the coefficient for GDM is 0.19 (an increase of $19 \%$ of the risk with optimal GWG). This phenomenon has also been described by preceding authors [7-9], and in fact, it might be an "optical" or a "perspective" bias due to our retrospective data. Li et al. [7] proposed an explanation which may be the good one: because the diagnosis of GDM occurs primarily at 26-28 weeks of gestation, treatment with diet and/or insulin plus increased physical activity may affect subsequent weight gain resulting in decreased weight gain in late pregnancy. This is emphasized in a recent paper in the United Kingdom (UPBEAT study): obese women with a positive OGTT at 27 weeks, and afterward a strong follow-up until delivery present lower weight gain than obesity considered as non-diabetic [10]. 
NON OBESE WOMEN BMI $<25 \mathrm{~kg} / \mathrm{m}^{2}, \mathrm{~N}=36,167$ (average GWG 11 to $23 \mathrm{~kg}$ )

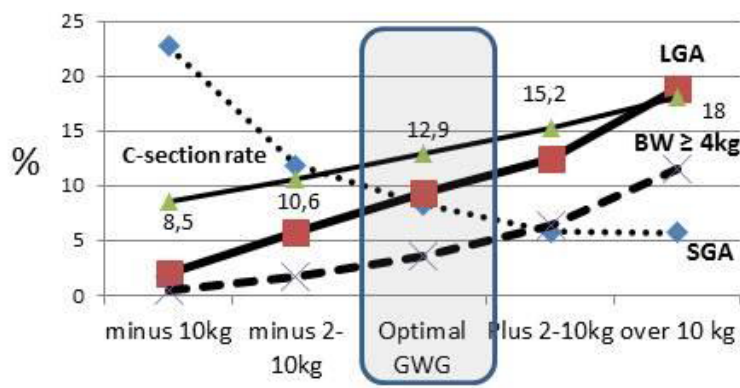

OBESITY CLASS I. 30-34,9 kg/ $\mathrm{m}^{2} . \mathrm{N}=6232$ (average GWG 0 to $6 \mathrm{~kg}$ )

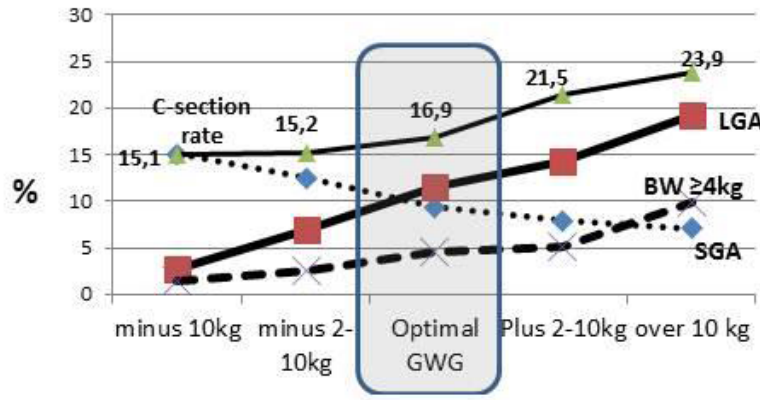

Overweight women $25-29,9 \mathrm{~kg} / \mathrm{m}^{2}, \mathrm{~N}=12,701$ (average GWG $5-11 \mathrm{~kg}$ )

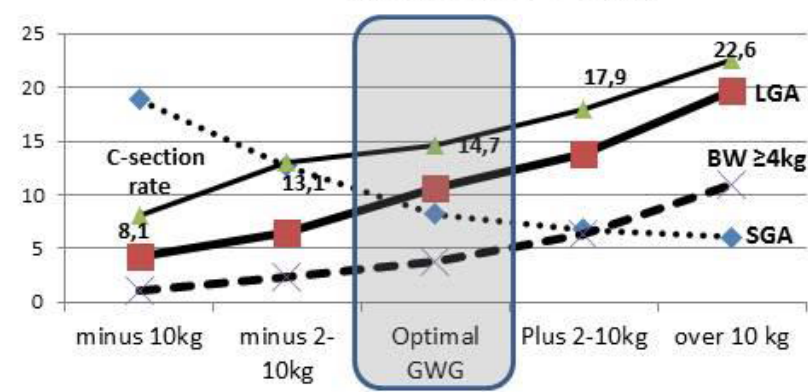

Severe Obese $\geq 35 \mathrm{~kg} / \mathrm{m}^{2} . \mathrm{N}=4071$ (average GWG 0 kg or loss of weight)

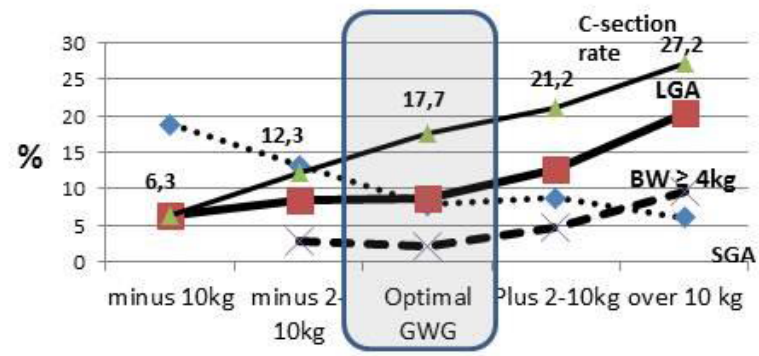

Figure 2: Simulation from the perspective of optimal or non-optimal GWG. Women having achieved their personal optGWG vs. moderately or severe insufficient or excessive GWG.

Indeed, it is because our study is retrospective, and that we mix the concept of optimal weight gain which could be theoretically known since the beginning of pregnancy with the diagnosis of GDM which is made much later: 24-28 weeks. If we did a prospective follow-up of obese pregnancies since the beginning of counselling to the woman, a moderate GWG (or even a loss of weight in severe obesities), incidence of GDM being quasi-parallel and proportional with the increase of BMI $[10,11]$, we predict that women would reach the $24^{\text {th }}$ week of gestation with lower rates of GDM diagnoses in a prospective management of such pregnancies.

Third: applicability of our linear equation for pragmatic management of future pregnancies elsewhere. The fact that the Maternal Fetal Corpulence Symbiosis (MFCS) has been achieved as a mathematical linear equation, it implies that it is also similar elsewhere. But, we do not feel fair to state that our formula, designed in our Reunionese population, would be adapted everywhere $[1,2]$. MFCS is based on the $10 \%$ crossing point of SGA-LGA. Therefore, these SGA/LGA definitions are different in different ethnicities (e.g. Eastern Asians, India, Africa etc...).

Let us consider the problem of SGA: for us, in Reunion island, being SGA at term is to be approximately less than 2500g. But, in India, the physiological SGA birthweight at term is $2200 \mathrm{~g}[12,13]$. This may be also in line with a recent WHO study arguing that definition of low-birthweight should be different $(<2200 \mathrm{~g}$ in Africa, $<2100 \mathrm{~g}$ in Asia, $<2200 \mathrm{~g}$ in Latin America) between different populations, and no more the universal below 2500g [14].
Considering the mothers, and countries like India or Japan which have a high rate of lean women [12-15]. In our formula, lean women of $18.5 \mathrm{~kg} / \mathrm{m}^{2}$ should have an optGWG of $20 \mathrm{~kg}$ (instead of $12.5-18$ $\mathrm{kg}$, IOM 2009 recommendations), but we do not feel that counselling a great proportion of Indian or Japenese women to gain $20 \mathrm{~kg}$ in their pregnancies before knowing their newborns' SGA-LGA rates is legitimate.

Considering now the problem of LGA: for example, macrosomic newborns with birthweights $\geq 4000 \mathrm{~g}$, in Reunion represent $3.9 \%$ of term babies, but it is $0.5 \%$ in India, $6.9 \%$ in China, $2.0 \%$ in Niger, $2.2 \%$ in Thailand, $9.3 \%$ in Paraguay $1.3 \%$ in Philippines, Nepal, Sri-Lanka etc...[16].

Therefore and logically, an Indian, Japenese, Chinese or Swedish linear equation should then be slightly different than ours $(y=-1.2 x$ + 42). This has been recently stated by Guan et al.: "There are specific Chinese birthweight curves for neonates. Therefore, with knowledge of the 10th percentile (SGA) and the 90th percentile (LGA) of newborns, we could test the proposed 'maternal-fetal-corpulence symbiosis', which was recently proposed ...." [3]. It is time and urgent to verify and establish in all continents the specific MFCS linear equation, to make it accessible everywhere on smartphones for health workers and women themselves [2,17]. Knowing the specific SGA-LGA definitions of newborns in a setting or a country, allows to easily find the MFCS curve everywhere.

Forth, the problem of macrosomia ( $\geq 4 \mathrm{~kg}$ ). These newborns are well-known to present a 10 fold higher risk of the fearsome shoulder 
dystocia [16]. The limit of $4 \mathrm{~kg}$ is considered to be the point where significant morbidities at delivery may occur [18], moreover, in the case of associated GDM [19]. Our simulation suggests that optGWG could lower very significantly the rate of macrosomia at birth: OR $0.75, \mathrm{p}=0.03$ in class 1 obesity, OR $0.46, \mathrm{P}=0.001$ in severely obese women (Tables 4 and 5) and, for all overweight women adjusted OR $0.67, \mathrm{p}<0.0001$, Table 6 . There is a strong current ongoing consensus on obesity, GWG, and consequences for maternal-fetal health. Urgent further work is required to identify ways to assist women in achieving an optimal GWG, with further RCT to confirm that such interventions would translate in a marked reduction in maternal/fetal morbidities, especially for macrosomia.

We do not comment here on the significant decrease of term preeclampsia, with high potential consequences also in health-costs policies. It has just been recently published in a specific study [6].

We have also tested intrauterine fetal deaths (IUFD, bottom of Table 1) as a meta-analysis showed that maternal obesity increases the risk for fetal deaths (OR 1.21 [1.09-1.35]), [20]. For the entire cohort, we did not find any difference between optGWG women and controls (Table 1) [21]. Finally, our results seem in contradiction of the large number of clinical trials, meta-analyses and individual participant data meta-analyses that have robustly shown that at best, dietary and lifestyle interventional studies have reduced GWG by $0.7 \mathrm{~kg}$ or $3.7 \mathrm{~kg}$ (minus the IOM recommendations for obese women $5-9 \mathrm{~kg}$ ) and had no effect on other pregnancy and birth outcomes including GDM, PE, PIH, LGA and macrosomic infants [22-25]. We have shown previously in our population that the IOM recommendations are correct for normal weight and overweight women [1], but not for obese, moreover if splitting them in Class I to III. Beginning at class II, women should even lose weight to achieve the SGA/LGA crossing point ("maternal fetal corpulence symbiosis", MFCS) [1]. All these studies [22-25] considered obesity as a whole $\left(\geq 30 \mathrm{~kg} / \mathrm{m}^{2}\right)$, while it seems more and more evident for many scholars that obesities class I, class II, and class III are somehow very different worlds concerning maternal/fetal morbidities.

The strength of our study is the capturing of all perinatal outcomes in a population of the area (ap. 360,000 inhabitants, and 5,000 births per year. With 4,300 births per year, the university maternity represents $85 \%$ of all births in the south of the island, all receiving level 3, European standard of care. The data in this large cohort are homogeneous as they were collected in a single center (no intercenter variability) and not based on national birth registers but directly from medical records (avoiding inadequate codes). The obvious weakness is the retrospective nature of this study, especially with the above discussion on GDM, demonstrating an association and not necessarily causation but we sincerely hope that our observations will trigger proper prospective trials because the potential health care benefits are immense.

\section{Conclusion}

We can help to actively counterbalance the morbid effects of high BMIs by individualized counselling for women on their GWG and have significant health (and cost) benefits with lowering c-section, term preeclampsia, low birthweights, and macrosomia rates. We renew our prediction that it should be also beneficial for gestational diabetes mellitus, but it can be verified only with a prospective study beginning since the first prenatal visit. We may have much to win from reducing weight gain during pregnancy in overweight/obese women. It is urgent to verify and establish in all continents the specific linear-curve of optGWG of each geographical/ethnic area, to make it accessible everywhere on smartphones for health workers and women themselves [2].

Disclosure of interest: All the authors attest that no conflict of interest exists regarding this work.

Contribution to authorship: All authors participated equally to this work and writings of the manuscript.

Ethics approval: This study was conducted in accordance with French legislation. As per new French law applicable to trials involving human subjects (Jardé Act), a specific approval of an ethics committee (comité de protection des personnes- CPP) is not required for this non-interventional study based on retrospective, anonymized data of authorized collections and written patient consent is not needed. Nevertheless, the study was registered on UMIN Clinical Trials Registry (identification number is UMIN000037012).

Funding: No special funding besides the normal existence of the South-Reunion perinatal database since 2001.

\section{References}

1. Robillard PY, Dekker G, Boukerrou M, Le Moullec N, Hulsey TC (2018) Relationship between pre-pregnancy maternal BMI and optimal weight gain in singleton pregnancies. Heliyon 4: e00615. [crossref]

2. Gestational weight gain calculator (English version) on smart phone. REPERE. RE (Reseau Perinatal REunion).

3. Guan P, Tang F, Sun G, Ren W (2019) Effect of maternal weight gain according to the Institute of Medicine recommendations on pregnancy outcomes in a Chinese population. J Int Med Res 47: 4397-4412. [crossref]

4. IOM (2009) Weight gain during pregnancy: reexamining the Guidelines. Institute of Medicine (US), National Research Council (US), Committee to Reexamine IOM Pregnancy Weight Guidelines. [crossref]

5. Robillard PY, Dekker G, Scioscia M, Bonsante F, Iacobelli S, et al. (2019) Increased BMI has a linear association with late-onset preeclampsia: A population-based study. PLoS One 14: e0223888. [crossref]

6. Robillard PY, Dekker GA, Boukerrou M, Boumahni B, Hulsey TC, et al. Optimizing gestational weight gain may halve the rate of late onset preeclampsia in overweight/ obese women: a retrospective analysis on 57,000 singleton pregnancies, Reunion Island. BMJ Open in Press.

7. Li C, Liu Y, Zhang W (2015) Joint and Independent Associations of Gestational Weight Gain and Pre-Pregnancy Body Mass Index with Outcomes of Pregnancy in Chinese Women: A Retrospective Cohort Study. PLoS One 10: e0136850. [crossref]

8. Nohr EA, Vaeth M, Baker JL, Sørensen TIa, Olsen J, et al. (2008) Combined associations of prepregnancy body mass index and gestational weight gain with the outcome of pregnancy. Am J Clin Nutr 87: 1750-1759. [crossref]

9. Riskin-Mashiah S, Damti A, Younes G, Auslander R (2011) Pregestational body mass index, weight gain during pregnancy and maternal hyperglycemia. Gynecol Endocrinol 27: 464-467. [crossreef]

10. Atakora L, Poston L, Hayes L, Flynn AC, White SL (2020) Influence of GDM Diagnosis and Treatment on Weight Gain, Dietary Intake and Physical Activity in Pregnant Women with Obesity: Secondary Analysis of the UPBEAT Study. Nutrients 12: E359. [crossref] 
Pierre-Yves Robillard (2020) The Urgent Need to Optimize Gestational Weight in Overweight/Obese Women to Lower Maternal-Fetal Morbidities: A Retrospective Analysis on 59,000 Singleton Term Pregnancies

11. Spaight C, Gross J, Horsch A, Puder JJ (2016) Gestational Diabetes Mellitus. Endocr Dev 31: 163-178.

12. Kinare AS, Chinchwadkar MC, Natekar AS, Coyaji KJ, Wills AK, et al. (2010) Patterns of fetal growth in a rural Indian cohort and comparison with a Western European population: data from the Pune maternal nutrition study. J Ultrasound Med 29: 215-223. [crossref]

13. Sebastian T, Yadav B, Jeyaseelan L, Vijayaselvi R, Jose R (2015) Small for gestational age births among South Indian women: temporal trend and risk factors from 1996 to 2010. BMC Pregnancy Childbirth 15: 7. [crossref]

14. Laopaiboon M, Lumbiganon P, Rattanakanokchai S, Chaiwong W, Souza JP, et al. (2019) An outcome-based definition of low birthweight for births in low- and middleincome countries: a secondary analysis of the WHO global survey on maternal and perinatal health. BMC Pediatrics 19: 166. [crossref]

15. Shindo R, Aoki M, Yamamoto Y, Misumi T, Miyagi E, et al. (2019) Optimal gestational weight gain for underweight pregnant women in Japan. Sci Rep 9: 18129.

16. Koyanagi A, Zhang J, Dagvadorj A, Hirayama F, Shibuya K, et al. (2013) Macrosomia in 23 developing countries: an analysis of a multicountry, facility-based, crosssectional survey. Lancet 381: 476-483. [crossref]

17. Simkin P (2003) Maternal positions and pelves revisited. Birth 30: 130-132. [crossref]

18. Graafmans WC, Richardus JH, Borsboom GJ, Bakketeig L, Langhoff-Roos J, et al. (2002) EuroNatal working group. Birth weight and perinatal mortality: a comparison of "optimal" birth weight in seven Western European countries. Epidemiology 13: 569-574. [crossref]
19. Robillard PY, Boukerrou M, Bonsante F, Hulsey TC, Gouyon JB (2019) Neonatal outcomes of macrosomic newborns $(4000 \mathrm{~g}+)$ of diabetic and non diabetic mothers: a study of 1,391 newborns. Integr Gyn Obstet J 2: 1-4.

20. Aune D, Saugstad OD, Henriksen T, Tonstad S (2014) Maternal body mass index and the risk of fetal death, stillbirth, and infant death: a systematic review and metaanalysis. JAMA 311: 1536-1546. [crossref]

21. Hutcheon JA, Bodnar LM (2014) A systematic approach for establishing the range of recommended weight gain in pregnancy. Am J Clin Nutr 100: 701-707. [crossref]

22. Rogozińska E, Zamora J, Marlin N, Betrán AP, Astrup A, et al. (2019) Gestational weight gain outside the Institute of Medicine recommendations and adverse pregnancy outcomes: analysis using individual participant data from randomised trials. BMC Pregnancy Childbirth 19: 322. [crossref]

23. Dodd JM, Turnbull D, McPhee AJ, Deussen AR, Grivell RM, et al. (2014) Antenatal lifestyle advice for women who are overweight or obese: the LIMIT randomised trial. BMJ 348: g1285. [crossref]

24. Dodd JM, Deussen AR, Louise J (2019) A Randomised Trial to Optimise Gestational Weight Gain and Improve Maternal and Infant Health Outcomes through Antenatal Dietary, Lifestyle and Exercise Advice: The OPTIMISE Randomised Trial. Nutrients 11: 2911. [crossref]

25. The International Weight Management in Pregnancy (i-WIP) Collaborative Group (2017) Effect of diet and physical activity based interventions in pregnancy on gestational weight gain and pregnancy outcomes: meta-analysis of individual participant data from randomised trials. BMJ 358: j3119. [crossref]

\section{Citation:}

Robillard PY, Dekker G, Boukerrou M, Boumahni B, Hulsey TC, Scioscia M (2020) The Urgent Need to Optimize Gestational Weight in Overweight/Obese Women to Lower Maternal-Fetal Morbidities: A Retrospective Analysis on 59,000 Singleton Term Pregnancies. ARCH Women Health Care Volume 3(3): 1-9. 